\title{
Determination of dynamic gradient elasticity length scales
}

Giorgio Carta MEng, MSc

Former Graduate Student, University of Sheffield, Sheffield, UK

Terry Bennett MEng, PhD

RCUK Research Fellow, University of Sheffield, Sheffield, UK
Harm Askes MSc, PhD

Professor of Computational Mechanics, University of Sheffield, Sheffield, UK

\begin{abstract}
Wave dispersion is a widely recognised phenomenon that occurs when elastic waves propagate through a heterogeneous microstructured material; reflection and refraction of higher frequencies leads to an apparent reduction of the wave speed with frequency. Enhanced continua are frequently employed to capture this phenomenon efficiently. Numerical experiments are performed in this paper to establish a procedure for the determination of the length scale parameters used in dynamic gradient elasticity using spectral analysis. Suitable values of the length scale parameters are determined and verified for a one-dimensional laminated bar and for a two-dimensional chequerboard plate.
\end{abstract}

\section{Introduction}

The mechanical behaviour of heterogeneous materials may be simulated by explicitly modelling the individual material phases that make up the composite or by calculation of their effective properties. The use of effective properties, however, may lead to a loss of information of the material behaviour that is driven by its microstructure. Explicit modelling of the discrete material phases, while capturing microstructural effects, may lead to onerous computational demands, particularly where a material or structure needs to be modelled in two or three dimensions. To overcome these problems for modelling composites, enhanced continua have been employed.

This short paper focuses on the modelling of wave dispersion phenomena in composite materials using gradient elasticity. In gradient elasticity, the usual equations of motion are extended with additional spatial derivatives of the displacements and/or the accelerations; see for instance the landmark paper of Mindlin (1964), the simplified format suitable for static applications due to Aifantis and co-workers (Aifantis, 1992; Altan and Aifantis, 1992; $\mathrm{Ru}$ and Aifantis, 1993) and the renewed interest in dynamic applications in recent years (Chen and Fish, 2001; Metrikine and Askes, 2002; Papargyri-Beskou et al., 2009; Rubin et al., 1995; Wang and Sun, 2002). In gradient elasticity, simple parameters are used to incorporate microstructural effects into a model without the need to model explicitly individual material phases, thus enabling a significant reduction in computational overhead. The additional model parameters, for dimensional consistency, have the dimensions of length and are henceforth referred to as length scales.

A debate still exists as to whether the length scale parameters in gradient elasticity are simply model parameters or physically identifiable quantities. Irrespective of this debate, in order that the method may be used in a predictive manner, methods whereby the length scales may be identified need to be developed. In this paper, spectral analysis of waves propagating through a hetero- geneous material is used to fit the length scales of dynamic gradient elasticity.

A short introduction to theory of gradient elasticity in dynamics is given in Section 2. A noteworthy feature of this particular model is the use of both stiffness and inertial length scales.

Section 3 investigates the wave dispersion behaviour of a heterogeneous bar. A spectral analysis procedure for the determination of a bar's dispersion curve is outlined and then applied to a bar where the individual material constituents are modelled explicitly. The numerical results are compared with an analytical solution for the dispersive behaviour, which contains the gradient elasticity length scales; by making an assumption as to the nature of the stiffness length scale, the inertial length scale may be calculated.

The techniques employed in Section 3 are then extended to two dimensions in Section 4 to show that the length scales derived are capable of describing both the p-wave and s-wave dispersion characteristics of a chequerboard patterned plate.

\section{Gradient elasticity theory in dynamics}

The equations of motion of a multidimensional dynamic system in gradient elasticity are expressed as (Askes et al., 2007)

$$
\begin{aligned}
\quad \rho\left(\ddot{\boldsymbol{u}}_{i}-l_{m}^{2} \ddot{\boldsymbol{u}}_{i, m m}\right)= & \boldsymbol{C}_{i j k l} \\
& \times\left(\frac{\boldsymbol{u}_{k, j l}+\boldsymbol{u}_{l, j k}}{2}-l_{\mathrm{s}}^{2} \frac{\boldsymbol{u}_{k, j l m m}+\boldsymbol{u}_{l, j k m m}}{2}\right)
\end{aligned}
$$

where $\boldsymbol{u}$ is the displacement vector, $\rho$ is the mass density, $\boldsymbol{C}$ is the elastic fourth-order tensor, while superimposed dots and indices following commas denote time and space derivatives, respectively. If the material is assumed to be linear elastic and isotropic, the elastic tensor reads 


$$
\boldsymbol{C}_{i j k l}=\lambda \delta_{i j} \delta_{k l}+\mu \delta_{i k} \delta_{j l}+\mu \delta_{i l} \delta_{j k}
$$

where $\lambda$ and $\mu$ are Lamé's constants. This model is only applicable to isotropic media; extension of the theory to anisotropy has recently been performed (Gitman et al., 2010).

In Equation 1 both higher-order inertia and stiffness appear, thus the model is considered as dynamically consistent (Metrikine and Askes, 2002). In order to make Equation 1 dimensionally valid, the length scales $l_{\mathrm{s}}$ and $l_{\mathrm{m}}$ are introduced. The latter quantities are related to the inherent microstructure of the material. In particular, $l_{\mathrm{s}}$ can be given by

$$
\text { 2. } \quad l_{\mathrm{s}}=\frac{L}{\sqrt{12}}
$$

where $L$ indicates the size of the representative volume element of the material in statics (Gitman et al., 2005). A more thorough evaluation of the length scale $l_{\mathrm{m}}$ will be provided in Section 3 .

High-frequency waves will be apparently slower than lowfrequency waves (Metrikine and Askes, 2002) in this model if $l_{\mathrm{m}}$ is chosen to be greater than $l_{\mathrm{s}}$. This is as observed in discrete lattices, which are often taken as a reference case when analysing wave dispersion in gradient elasticity. Therefore, to make the gradient elasticity model described by Equation 1 physically reliable, it must be assumed that $l_{\mathrm{m}} \geqslant l_{\mathrm{s}}$.

\section{Dispersion curves of a heterogeneous bar}

The validity of the gradient elasticity theory described in Section 2 is verified by comparing the actual dispersion curves of a heterogeneous material, determined by modelling the heterogeneities explicitly, with the analytical dispersion curves provided by the gradient elasticity approach.

\subsection{Procedure to determine the dispersion curves of the explicitly modelled bar}

The actual dispersion curves of the material are obtained through a procedure commonly adopted in the spectral analysis of surface waves (SASW), which is a technique mainly used in geotechnical engineering to assess in situ properties of the soil layers (Kim and Park, 2002).

The SASW procedure consists of applying an impulsive load to the structure in order to produce the propagation of an infinite series of sinusoidal waves with different frequency. The acceleration responses at two distinct positions, called receivers, are captured in the time domain and subsequently converted into the frequency domain by using the fast Fourier transform. The latter algorithm provides the magnitudes $M$ and the phases $\theta$ of the two signals as functions of the frequency $f$. If evaluated between $0^{\circ}$ and $360^{\circ}$, the phases must be 'unwrapped', that is their values must be incremented by $360^{\circ}$ whenever they complete a $360^{\circ}$ angle. In this way, it is possible to compute the correct number of cycles that each sinusoidal wave possesses between the two receivers as

3. $n_{\text {cyc }}(f)=\frac{\Delta \theta_{\text {unwrapped }}(f)}{360^{\circ}}$

where $\Delta \theta$ represents the phase shift, namely $\Delta \theta=\theta_{1}-\theta_{2}$.

Thus, the phase velocity $c_{\mathrm{p}}$ and the wave number $k$ can be calculated with the following formulae

4. $\quad c_{\mathrm{p}}(f)=\frac{d}{n_{\mathrm{cyc}}(f) / f}$

5. $k(f)=\frac{2 \pi}{c_{\mathrm{p}}(f) / f}$

where $d$ denotes the distance between the two receivers. Finally, the dispersion curves are retrieved by plotting the values of the phase velocity against the values of the wave number for each frequency considered.

\subsection{Description of the one-dimensional model}

The laminated bar shown in Figure 1 is studied, which has previously been examined in previous works (Bennett et al., 2007; Chen and Fish, 2001).

The bar is made of two constituents with a volume fraction $\alpha=0 \cdot 5$, and consists of periodic cells of length $L=1 \mathrm{~m}$. Moreover, it is long enough to prevent the disturbance of the waves reflected at the fixed end when evaluating the response of the system to the external loads.

The laminate is first studied by numerically modelling the heterogeneities explicitly. It is discretised in space with a mesh

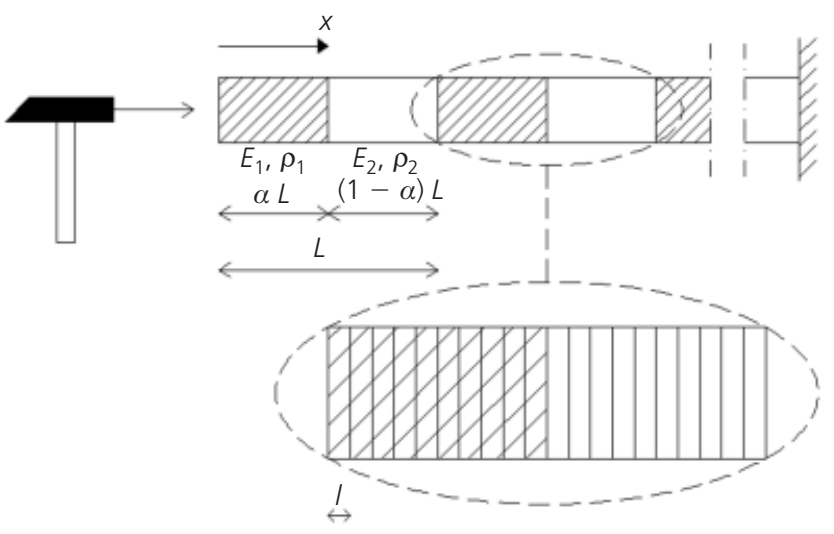

Figure 1. One-dimensional laminate made of two components 
consisting of linear elements with constant size $l=0 \cdot 1 \mathrm{~m}$. Newmark's constant-average-acceleration scheme is adopted, with a time step $\Delta t=0 \cdot 1 \mathrm{~s}$, enough to traverse one element per time step, to minimise numerical dispersion. The properties of the two materials are varied in order to change the dispersive characteristics of the structure. In particular, 14 distinct cases are considered, which are summarised in Table 1 , where $E_{i}, \rho_{i}$ and $c_{\mathrm{wav}, i}=\sqrt{E_{i} / \rho_{i}}$ denote the Young's modulus, the density and the wave speed respectively for material phase $i$.

The effective Young's modulus $E$ and density $\rho$ can be obtained from the following expressions (Chen and Fish, 2001)

$$
\text { 6. } E=\frac{E_{1} E_{2}}{(1-\alpha) E_{1}+\alpha E_{2}}
$$

7. $\rho=\alpha \rho_{1}+(1-\alpha) \rho_{2}$

For all cases $E=1 \mathrm{~N} / \mathrm{m}^{2}$ and $\rho=1 \mathrm{~kg} / \mathrm{m}^{3}$ was used.

\subsection{Comparison between the numerical and analytical dispersion curves}

The dispersion curves of the explicit numerical modelled laminate depicted in Figure 1 are determined numerically by using the SASW procedure described in Section 3.1. The analytical dispersion curves predicted by the gradient elasticity theory discussed in Section 2 reads (Askes et al., 2007)

8. $c_{\mathrm{p}}=c_{\mathrm{e}} \sqrt{\frac{1+l_{\mathrm{s}}^{2} k^{2}}{1+l_{\mathrm{m}}^{2} k^{2}}}$ where $c_{\mathrm{e}}=\sqrt{E / \rho}$ is the wave velocity in classical elasticity, which is equal to $1 \mathrm{~m} / \mathrm{s}$ for all the 14 cases presented in Table 1 .

The length scale $l_{\mathrm{s}}$ appearing in Equation 8 is calculated through Equation 2, from which it results that it is identical in all cases. The length scale $l_{\mathrm{m}}$ depends on the dispersive properties of the laminate. In particular, the more dispersive the material, the greater $l_{\mathrm{m}}$ should be. The values of the parameter $l_{\mathrm{m}}$ can be found by determining, for each case examined, the analytical dispersion curve that best fits the numerical data. They are reported in Table 1 and plotted in Figure 2, where they are normalised with respect to $l_{\mathrm{s}}$ and are related to the ratio between the wave velocities in the two constituents $c_{\mathrm{wav}, 1} / \mathcal{c}_{\mathrm{wav}, 2}$.

In Figure 3 four different cases are shown; for each of them, the

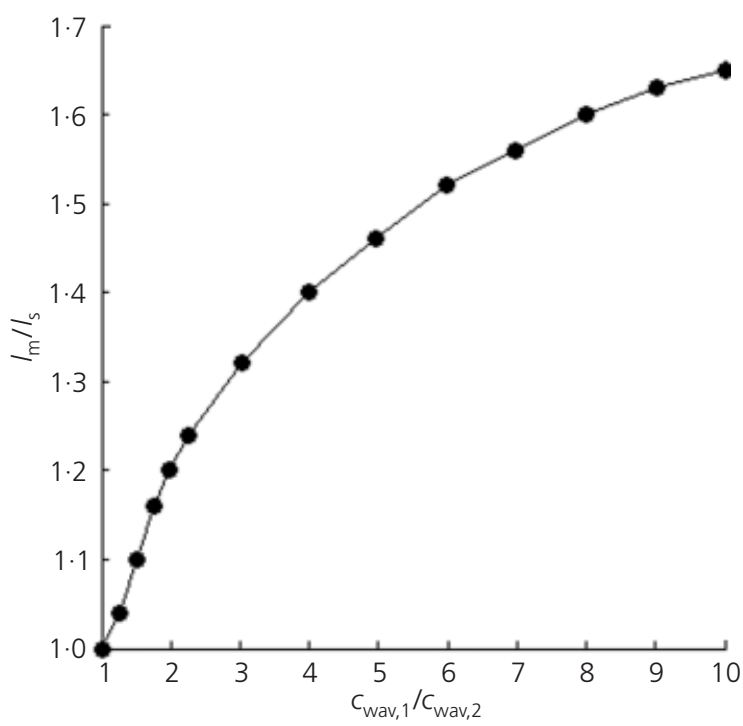

Figure 2. Values of $/ \mathrm{m} / I_{\mathrm{s}}$ plotted against the ratio $C_{\text {wav }, 1} / C_{\text {wav, } 2}$

\begin{tabular}{lcccccccc}
\hline Case & $E_{1}: \mathrm{N} / \mathrm{m}^{2}$ & $\rho_{1}: \mathrm{kg} / \mathrm{m}^{3}$ & $E_{2}: \mathrm{N} / \mathrm{m}^{2}$ & $\rho_{2}: \mathrm{kg} / \mathrm{m}^{3}$ & $C_{\text {wav, }, 1}: \mathrm{m} / \mathrm{s}$ & $C_{\text {wav }, 2}: \mathrm{m} / \mathrm{s}$ & $\frac{C_{\text {wav }, 1}}{C_{\text {wav }, 2}}$ & $I_{\mathrm{m}} / I_{\mathrm{s}}$ \\
\hline 1 & 1.00 & 1.0000 & 1.00 & 1.0000 & 1.00 & 1.00 & 1.00 & 1.00 \\
2 & 1.30 & 1.0100 & $8.13 \times 10^{-1}$ & 0.9900 & 1.13 & 0.91 & 1.25 & 1.04 \\
3 & 1.67 & 1.0200 & $7.14 \times 10^{-1}$ & 0.9800 & 1.28 & 0.85 & 1.50 & 1.10 \\
4 & 2.12 & 1.0300 & $6.54 \times 10^{-1}$ & 0.9700 & 1.43 & 0.82 & 1.75 & 1.16 \\
5 & 2.60 & 1.0400 & $6.19 \times 10^{-1}$ & 0.9600 & 1.58 & 0.80 & 2.00 & 1.20 \\
6 & 3.47 & 1.0800 & $5.84 \times 10^{-1}$ & 0.9200 & 1.79 & 0.80 & 2.25 & 1.24 \\
7 & 7.40 & 1.2000 & $5.36 \times 10^{-1}$ & 0.8000 & 2.48 & 0.82 & 3.00 & 1.32 \\
8 & $2.00 \times 10^{1}$ & 1.4200 & $5.13 \times 10^{-1}$ & 0.5800 & 3.75 & 0.94 & 4.00 & 1.40 \\
9 & $5.00 \times 10^{1}$ & 1.6000 & $5.05 \times 10^{-1}$ & 0.4000 & 5.59 & 1.12 & 5.00 & 1.46 \\
10 & $1.10 \times 10^{2}$ & 1.7200 & $5.02 \times 10^{-1}$ & 0.2800 & 8.00 & 1.34 & 6.00 & 1.52 \\
11 & $2.20 \times 10^{2}$ & 1.8000 & $5.01 \times 10^{-1}$ & 0.2000 & 11.06 & 1.58 & 7.00 & 1.56 \\
12 & $4.60 \times 10^{2}$ & 1.8700 & $5.01 \times 10^{-1}$ & 0.1300 & 15.68 & 1.96 & 8.00 & 1.60 \\
13 & $1.12 \times 10^{3}$ & 1.9300 & $5.00 \times 10^{-1}$ & 0.0700 & 24.09 & 2.67 & 9.00 & 1.63 \\
14 & $1.00 \times 10^{6}$ & 1.9999 & $5.00 \times 10^{-1}$ & 0.0001 & 707.12 & 70.71 & 10.00 & 1.65
\end{tabular}

Table 1. Material properties and corresponding values of $/ \mathrm{m} / \mathrm{s}_{\mathrm{s}}$ 


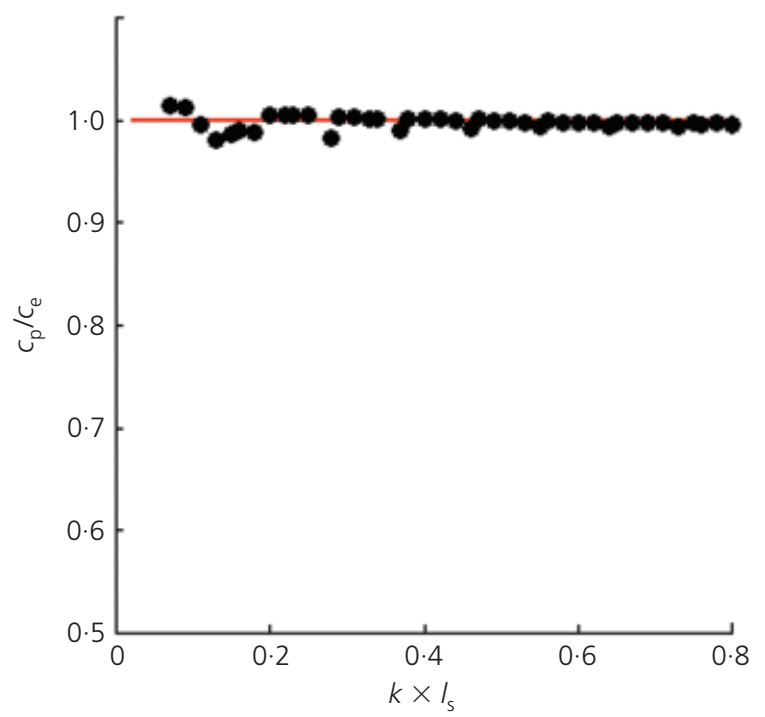

(a)

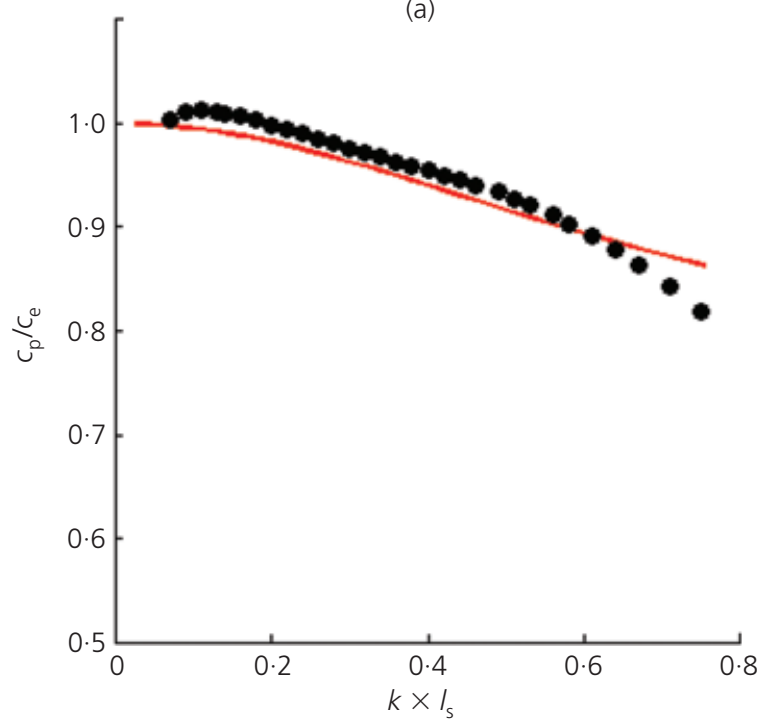

(c)

Figure 3. Comparison between the numerical (dotted lines) and analytical (solid lines) dispersion curves for four cases presented in Table 1: (a) case 1; (b) case 4; (c) case 8; (d) case 14

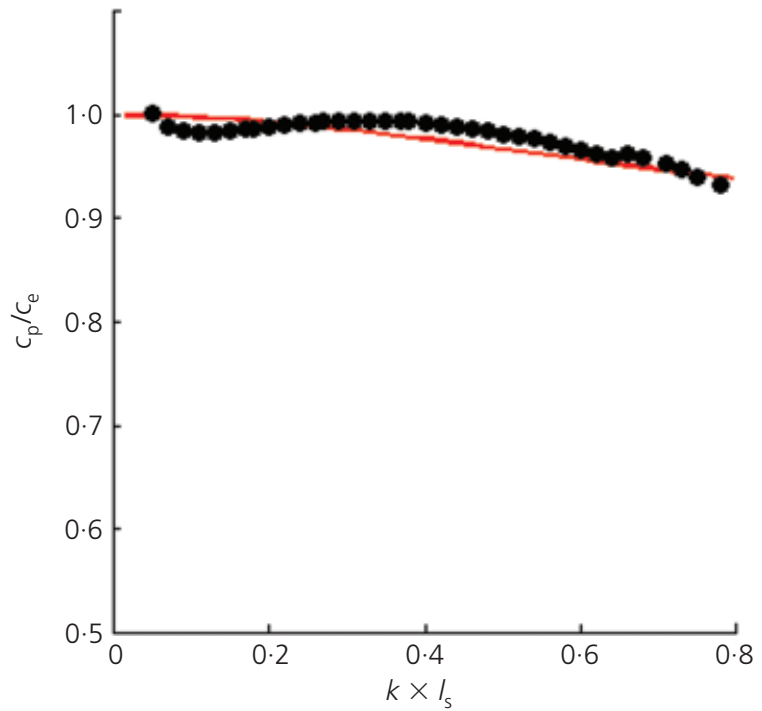

(b)

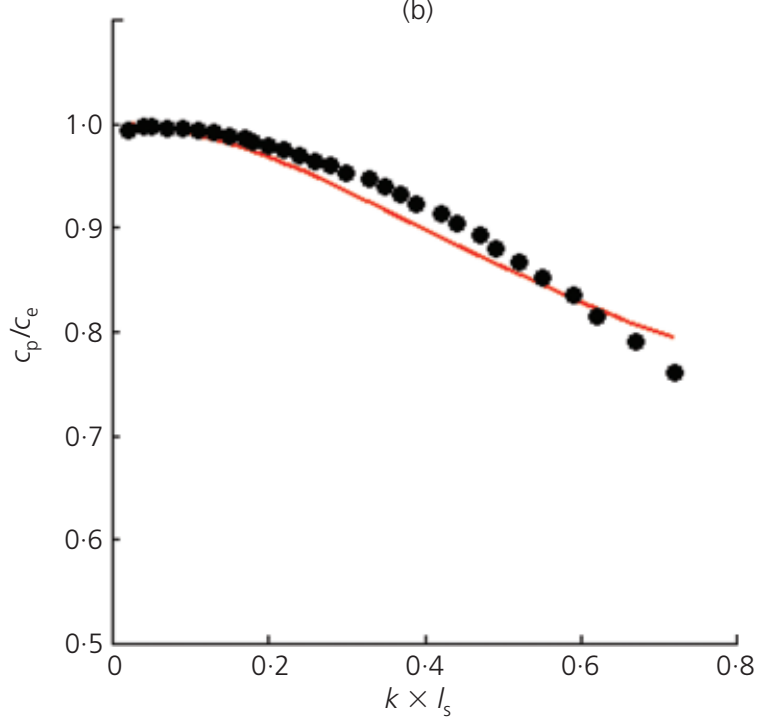

(d) dotted line represents the explicit numerical dispersion curve, while the solid line indicates the gradient analytical dispersion curve derived from Equation 8. It is apparent from Figure 3 that the gradient analytical approach gives a very good approximation of the explicit numerical dispersion curves of the material, with a maximum error always less than 5\%. These results thus show the validity of the gradient analytical approach in the onedimensional case as far as the dispersive behaviour of heterogeneous media is concerned.

\section{Dispersion curves of a heterogeneous plate}

In this section, a two-dimensional model is studied in order to verify the validity of the gradient elasticity formulation in describing the dispersive behaviour of heterogeneous materials in more than one spatial dimension.

\subsection{Description of the two-dimensional model}

The two-dimensional model under consideration is a plate made of two constituents disposed in a chequerboard pattern, as shown in Figure 4. The plate is fixed on one side and is subjected on the other side to impulsive loads, one directed parallel to the $x$ axis, which generates compressive waves, and the other one acting along the $y$ direction, which produces shear waves. The response of the external excitations is determined far enough from the fixed end in order to avoid the interference of the reflected waves.

The properties of the two materials are summarised in Table 2 . 
Determination of dynamic gradient

elasticity length scales

Carta, Bennett and Askes

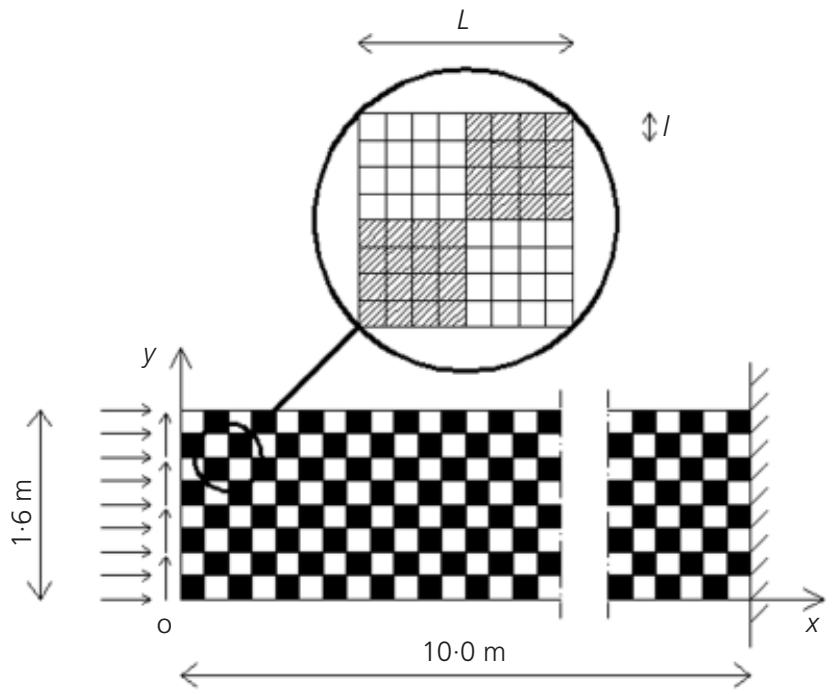

Figure 4. Plate consisting of two materials forming a chequerboard structure

More specifically, six distinct cases are taken into account, whereby all the properties are the same apart from the density of material $2\left(\rho_{2}\right)$, which is varied in order to assign different dispersive features to the plate.

The model consists of periodic cells of length $L=0.4 \mathrm{~m}$. It is meshed with square quadrilateral elements of constant size $l=0.05 \mathrm{~m}$, with plain strain conditions assumed.

\subsection{Compressive wave dispersion}

When the plate is excited by the impulsive load acting along the $x$ direction (see Figure 4), compressive waves propagate through the structure. The real dispersion curves of the material are again obtained by applying the SASW procedure described in Section 3.1. The explicit numerical dispersion curves corresponding to cases 3 and 6 considered in Table 2 are plotted in Figure 5, where they are indicated by dots. In the latter figure, $C$ denotes the phase velocity, while $K$ represents the modulus of the wave vector in two dimensions, given by $K=\sqrt{k_{x}^{2}+k_{y}^{2}}$.

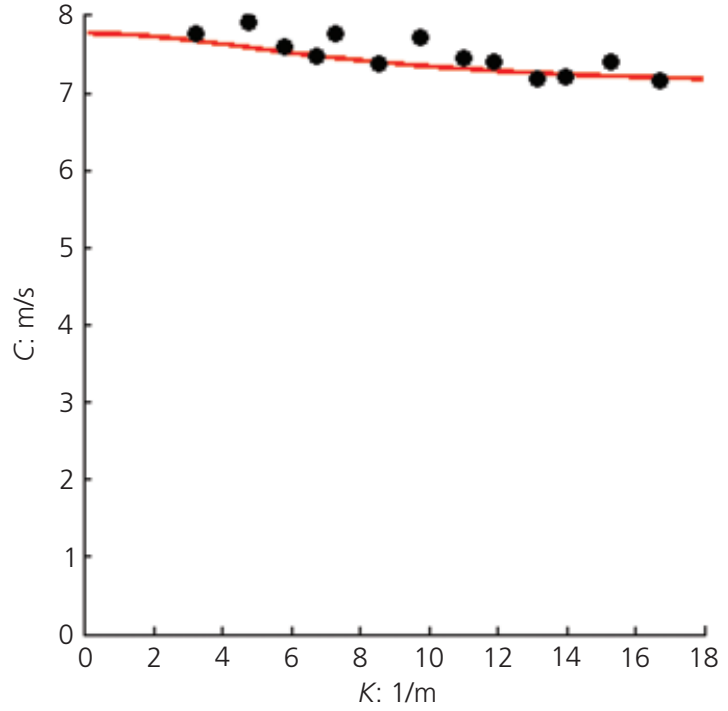

(a)

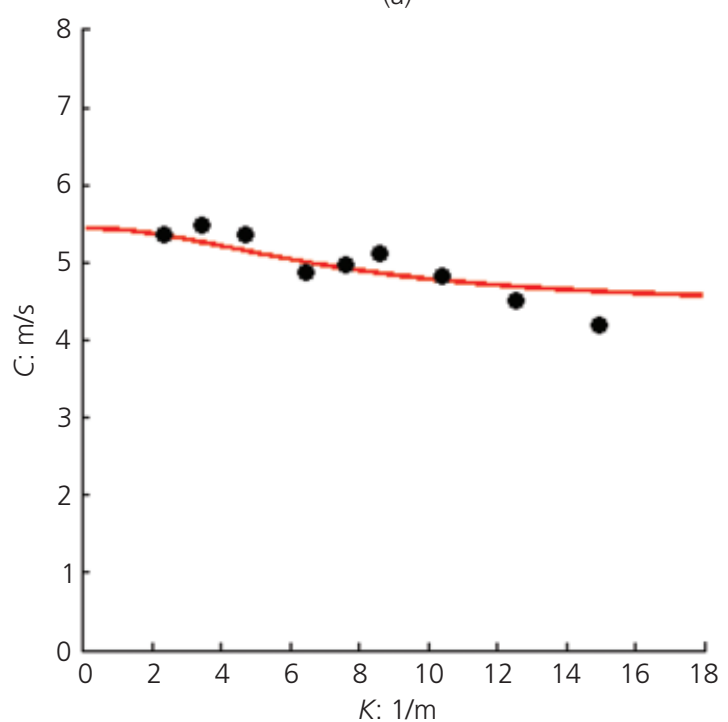

(b)

Figure 5. Comparison between the numerical (dots) and analytical (solid lines) dispersion curves for compressive waves: (a) case 3;

(b) case 6

\begin{tabular}{|c|c|c|c|c|c|c|c|}
\hline Case & $E_{1}=E_{2}: \mathrm{N} / \mathrm{m}^{2}$ & $\rho_{1}: \mathrm{kg} / \mathrm{m}^{3}$ & $\rho_{2}: \mathrm{kg} / \mathrm{m}^{3}$ & $v_{1}=v_{2}$ & $C_{\text {wav }, 1}: \mathrm{m} / \mathrm{s}$ & $c_{\text {wav }, 2}: \mathrm{m} / \mathrm{s}$ & $\frac{C_{\text {wav }, 1}}{C_{\text {wav }, 2}}$ \\
\hline 1 & $2.20 \times 10^{5}$ & 2500 & 2500 & 0.2 & $9 \cdot 4$ & $9 \cdot 4$ & 1.00 \\
\hline 2 & $2.20 \times 10^{5}$ & 2500 & 3900 & 0.2 & $9 \cdot 4$ & $7 \cdot 5$ & $1 \cdot 25$ \\
\hline 3 & $2.20 \times 10^{5}$ & 2500 & 5600 & $0 \cdot 2$ & $9 \cdot 4$ & $6 \cdot 3$ & $1 \cdot 50$ \\
\hline 4 & $2.20 \times 10^{5}$ & 2500 & 7700 & 0.2 & $9 \cdot 4$ & $5 \cdot 3$ & 1.75 \\
\hline 5 & $2.20 \times 10^{5}$ & 2500 & 10000 & $0 \cdot 2$ & $9 \cdot 4$ & $4 \cdot 7$ & $2 \cdot 00$ \\
\hline 6 & $2.20 \times 10^{5}$ & 2500 & 12700 & 0.2 & $9 \cdot 4$ & $4 \cdot 2$ & $2 \cdot 25$ \\
\hline
\end{tabular}

Table 2. Material properties of the plate depicted in Figure 4 
On the other hand, the solid lines in Figure 5 represent the gradient analytical dispersion curves provided by the gradient elasticity theory (Bennett and Askes, 2009)

9. $C=C_{\mathrm{p}} \sqrt{\frac{1+l_{\mathrm{s}}^{2} K^{2}}{1+l_{\mathrm{m}}^{2} K^{2}}}$

where $C_{\mathrm{p}}$ is the velocity of the compressive waves with infinite wavelength, given by

10. $C_{\mathrm{p}}=\sqrt{\frac{E(1-v)}{\rho(1+v)(1-2 v)}}$

The parameters $E, \rho$ and $v$ denote the effective properties of the plate. The effective density $\rho$ is calculated through Equation 7; the values of the effective Young's modulus $E$ and of the effective Poisson ratio $v$ coincide with those of the two components. The length scales $l_{\mathrm{s}}$ and $l_{\mathrm{m}}$ that appear in Equation 9 are determined as from Equation 2 and Table 1, respectively.

Figure 5 shows that, also in two dimensions, the gradient elasticity formulation is capable of predicting accurately the explicit numerical dispersion curves of the material. However, by comparing Figure 5 with Figure 3, it is apparent that in two dimensions the scattering of the numerical data is more evident than in the one-dimensional case.

\subsection{Shear wave dispersion}

Shear waves are generated if the plate shown in Figure 4 is subjected to the impulsive load directed along the $y$ axis. The explicit numerical and gradient analytical dispersion curves for cases 3 and 6 presented in Table 2 are plotted in Figure 6 as dots and solid lines, respectively.

The gradient analytical dispersion curves predicted by the gradient elasticity formulation are given by (Bennett and Askes, 2009)

11

$$
C=C_{\mathrm{s}} \sqrt{\frac{1+l_{\mathrm{s}}^{2} K^{2}}{1+l_{\mathrm{m}}^{2} K^{2}}}
$$

where $C_{\mathrm{s}}$ is the velocity of the shear waves with infinite wavelength, expressed by

12.

$$
C_{\mathrm{s}}=\sqrt{\frac{E}{2 \rho(1+v)}}
$$

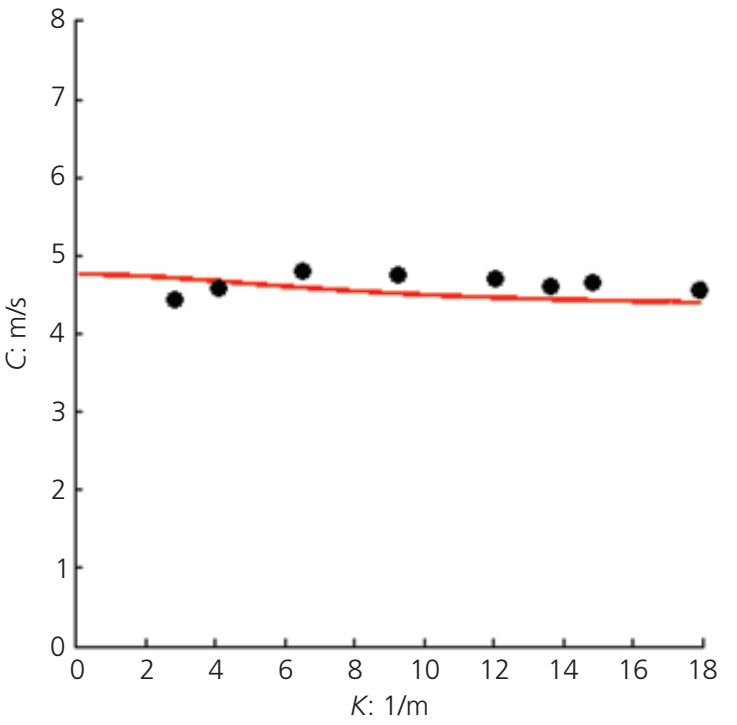

(a)

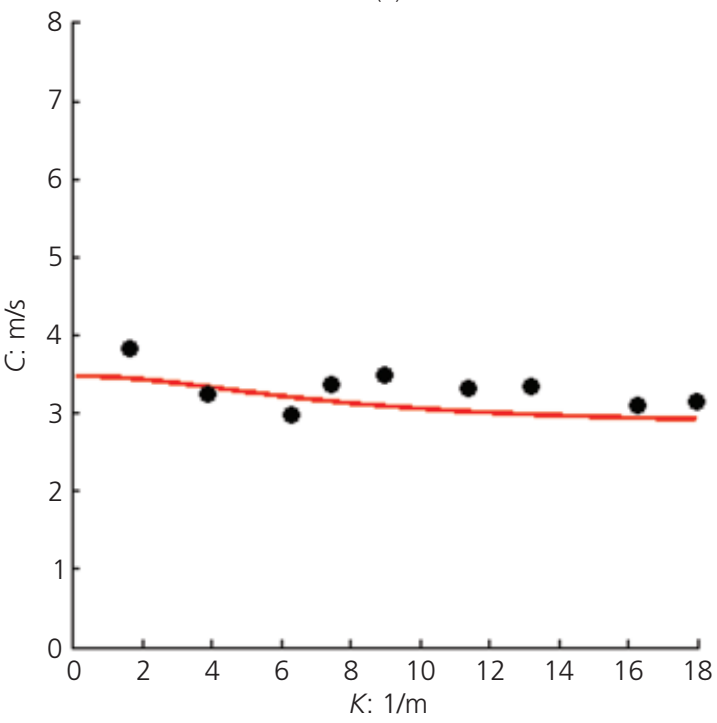

(b)

Figure 6. Comparison between the numerical (dots) and analytical (solid lines) dispersion curves for shear waves: (a) case 3;

(b) case 6

where the length scales $l_{\mathrm{s}}$ and $l_{\mathrm{m}}$ are calculated with Equation 2 and Table 1, respectively. The comparison between Equations 9 and 11 shows that the gradient analytical dispersion curves relative to compressive and shear waves have the same form, the only difference being the constant by which they are scaled.

Figure 6 illustrates that where shear waves are concerned, the actual dispersion curves of the material are fitted well by the gradient analytical approach.

\section{Conclusions}

Gradient elasticity can provide an effective and efficient tool for the modelling of composite materials. A potential drawback of 
Determination of dynamic gradient

elasticity length scales

Carta, Bennett and Askes gradient elasticity is the identification of the model length scale parameters. To address this, a spectral analysis procedure has been employed to identify the inertial length scale parameter used in a gradient elasticity formulation for the modelling of dispersive wave propagation behaviour in composite materials. While the procedure employed cannot a priori identify the model parameters, a simple test procedure is developed whereby the inertial length scale can easily be identified.

A clear relationship between the ratio of the stiffness and inertial length scales and the ratio of the elastic wave speeds in the two constituent materials can be identified for a given microstructural geometry. The current work is restricted to a simple test geometry; however, the extension of the technique to general geometries is the basis for further work. Likewise, the extension to composites comprising more than two phases remains a challenge.

\section{REFERENCES}

Aifantis EC (1992) On the role of gradients in the localization of deformation. International Journal of Engineering Science 30(10): 1279-1299.

Altan SB and Aifantis EC (1992) On the structure of the mode III crack-tip in gradient elasticity. Metallurgica et Materialia 26(2): 319-324.

Askes H, Bennett T and Aifantis EC (2007) A new formulation and $\mathrm{C}^{0}$ implementation of dynamically consistent gradient elasticity. International Journal of Numerical Methods in Engineering 72(1): 111-126.

Bennett T and Askes H (2009) Finite element modelling of wave dispersion with dynamically consistent gradient elasticity. Computational Mechanics 43(6): 815-825.

Bennett T, Gitman IM and Askes H (2007) Elasticity theories with higher-order gradients of inertia and stiffness for the modelling of wave dispersion in laminates. International Journal of Fracture 148(2): 185-193.
Chen W and Fish J (2001) A dispersive model for wave propagation in periodic heterogeneous media based on homogenization with multiple spatial and temporal scales. Transactions ASME, Journal of Applied Mechanics 68(2): 153-161.

Gitman IM, Askes $\mathrm{H}$ and Aifantis EC (2005) The representative volume size in static and dynamic micro-macro transitions. International Journal of Fracture 135(1-4): L3-L9.

Gitman IM, Askes H, Kuhl E and Aifantis EC (2010) Stress concentrations in fractured compact bone simulated with a special class of anisotropic gradient elasticity. International Journal of Solids and Structures 47(9): 1099-1107.

Kim DS and Park HC (2002) Determination of dispersive phase velocities for SASW method using harmonic wavelet transform. Soil Dynamics and Earthquake Engineering 22(8): 675-684

Metrikine AV and Askes H (2002) One-dimensional dynamically consistent gradient elasticity models derived from a discrete microstructure. Part 1: Generic formulation. European Journal of Mechanics A/Solids 21(4): 573-588.

Mindlin RD (1964) Micro-structure in linear elasticity. Archive for Rational Mechanics and Analysis 16(1): 51-78.

Papargyri-Beskou S, Polyzos D and Beskos DE (2009) Wave dispersion in gradient elastic solids and structures: a unified approach. International Journal of Solids and Structures 46(21): 3751-3759.

Ru CQ and Aifantis EC (1993) A simple approach to solve boundary-value problems in gradient elasticity. Acta Mechanica 101(1-4): 59-68.

Rubin MB, Rosenau P and Gottlieb O (1995) Continuum model of dispersion caused by inherent material characteristic length. Journal of Applied Physics 77(8): 4054-4063.

Wang Z-P and Sun CT (2002) Modeling micro-inertia in heterogeneous materials under dynamic loading. Wave Motion 36(4): 473-485.

\section{WHAT DO YOU THINK?}

To discuss this paper, please email up to 500 words to the editor at journals@ice.org.uk. Your contribution will be forwarded to the author(s) for a reply and, if considered appropriate by the editorial panel, will be published as a discussion in a future issue of the journal.

Proceedings journals rely entirely on contributions sent in by civil engineering professionals, academics and students. Papers should be 2000-5000 words long (briefing papers should be 1000-2000 words long), with adequate illustrations and references. You can submit your paper online via www.icevirtuallibrary.com/content/journals, where you will also find detailed author guidelines. 